\title{
Avaliação da assistência pré-natal às gestantes de Vitória da Conquista - Bahia
}

\author{
Evaluation of prenatal care for pregnant women in Vitória da Conquista - Bahia \\ Evaluación de la atención prenatal a gestantes en Vitória da Conquista - Bahia
}

Ana Paula Barros Poloni ${ }^{1 *}$, Michela Macedo Lima Costa ${ }^{1}$, Aline Benevides Sá Feres ${ }^{1}$, Karine Brito Matos Santos ${ }^{1}$, Bárbara Dielly Costa Balisa ${ }^{1}$, Daniel Thales Souza Santos ${ }^{1}$, Shesllen Mikaelly Cruz Corrêa ${ }^{1}$, Mariana Louzada Bittencourt ${ }^{1}$, Rebeca Guariento Rezende ${ }^{1}$, Ricely Almeida Rezende ${ }^{1}$.

\section{RESUMO}

Objetivo: Avaliar a assistência pré-natal ofertada às gestantes de Vitória da Conquista - Bahia durante o ano de 2019. Métodos: Foi realizado um estudo de caráter descritivo, com natureza e abordagem quantitativa. Os dados secundários analisados derivam de prontuários eletrônicos que alimentam o sistema e-SUS AB. Resultados: Observou-se que 3,5\% das gestantes não realizaram nenhuma consulta pré-natal, $7,5 \%$ realizaram de uma a três consultas, 30,93\% realizaram de quatro a seis consultas e 58,42\% realizaram sete ou mais consultas. A média de consultas por gestante foi de 5,77. Os exames mais solicitados foram exame de urina $(91,91 \%)$ e hemograma $(91,69 \%)$, e os menos solicitados foram teste rápido para HIV $(2,54 \%)$, tipagem sanguínea $(20,93 \%)$ e pesquisa do fator $\mathrm{RH}(23,03 \%)$. A porcentagem de consultas puerperais efetuadas atingiu 23,78\%. Conclusão: A assistência pré-natal às gestantes de Vitória da Conquista apresentou baixa adequação. Não houve acompanhamento pré-natal a todas as mulheres grávidas, a média de consultas por mulher foi abaixo do indicado, e a realização dos exames básicos do pré-natal e da consulta puerperal apresentam defasagens.

Palavras-chave: Assistência pré-natal, Atenção básica, Pré-natal, Gestante.

\begin{abstract}
Objective: Evaluate the prenatal care offered to pregnant women in Vitória da Conquista - Bahia during 2019. Methods: A descriptive study was carried out, with a quantitative approach and nature. The secondary data analyzed are derived from electronic medical records that feed the e-SUS AB system. Results: It was observed that $3.5 \%$ of pregnant women did not have any prenatal consultations, $7.5 \%$ had one to three consultations, $30.93 \%$ had four to six consultations and $58.42 \%$ had seven or more consultations. The average number of consultations per pregnant woman was 5.77. The most requested tests were urine tests $(91.91 \%)$ and blood count (91.69\%), and the least requested tests were a rapid HIV test (2.54\%), blood typing (20.93\%) and $\mathrm{RH}$ factor $(23.03 \%)$. The percentage of postpartum consultations carried out reached $23.78 \%$. Conclusion: Prenatal care for pregnant women in Vitória da Conquista showed low adequacy. There was no prenatal follow-up for all pregnant women, the average number of consultations per woman was lower than indicated, and the performance of basic prenatal examinations and postpartum consultations are lagging.
\end{abstract}

Key words: Prenatal care, Basic attention, Prenatal, Pregnant.

\section{RESUMEN}

Objetivo: Evaluar la atención prenatal ofrecida a las gestantes en Vitória da Conquista - Bahía durante el 2019. Métodos: Se realizó un estudio descriptivo, con abordaje cuantitativo y de naturaleza. Los datos secundarios analizados se derivan de historias clínicas electrónicas que alimentan el sistema e-SUS AB. Resultados: Se observó que el 3,5\% de las embarazadas no tuvo ninguna consulta prenatal, el 7,5\% tuvo de una a tres consultas, el $30,93 \%$ tuvo de cuatro a seis consultas y el $58,42 \%$ tuvo siete o más consultas. El promedio de consultas por gestante fue de 5,77 . Las pruebas más solicitadas fueron pruebas de orina $(91,91 \%)$ y hemograma $(91,69 \%)$, y las pruebas menos solicitadas fueron una prueba rápida de $\mathrm{VIH}(2,54 \%)$,

1 Faculdade Santo Agostinho (FASA), Vitória da Conquista - BA. *E-mail: anapaulabpoloni@gmail.com 
tipado sanguíneo (20,93\%) y factor $\mathrm{RH}(23,03 \%)$. El porcentaje de consultas posparto realizadas alcanzó el $23,78 \%$. Conclusión: La atención prenatal a gestantes en Vitória da Conquista mostró baja adecuación. No hubo seguimiento prenatal para todas las gestantes, el promedio de consultas por mujer fue menor al indicado y la realización de exámenes prenatales básicos y consultas posparto están rezagadas.

Palabras clave: Cuidado prenatal, Atención básica, Prenatal, Embarazada.

\section{INTRODUÇÃO}

A morbimortalidade materna está entre os principais indicadores de qualidade da saúde, além disso, esse indicador também consegue avaliar de forma indireta a qualidade da assistência prestada as mulheres gravidas, tanto durante a gestação como, também, durante o parto e puerpério. Apensar de ser um bom indicador, infelizmente muitas vezes os dados não são registados no sistema, por exemplo, na Estratégia de Informatização da Atenção Básica (e-SUS AB), assim, acabada por não ser tão fidedigno esses indicadores em saúde (FERREIRA TLDS, et al., 2017).

O processo de cuidado que envolve a mulher gestante e o neonato deve ser fundamentado na qualidade e humanização. O pré-natal, para se tornar efetivo, necessita de recursos disponíveis, organização e relações saudáveis, pautadas em princípios éticos, que garantam privacidade e autonomia a mulher e sua família. Além disso, a assistência ao pré-natal propõe-se orientar e esclarecer as possíveis dúvidas que as gestantes têm durante a gestão, buscando assim, dar mais conforto e segurança as grávidas (BRASIL, 2012).

O objetivo do acompanhamento pré-natal é garantir uma gestação e parto adequados, sem prejuízos à saúde da mãe, incluindo também aspectos psicossociais, ações educativas e preventivas. Também tem como objetivo reduzir a morbimortalidade materno-infantil, identificar os possíveis risco de problemas no desenvolvimento intrauterino e intervir a tempo, para que não haja atraso no desenvolvimento do feto e, por fim, reduzir o baixo peso dos recém-nascidos ao nascimentos. Infelizmente é um desafio, especialmente no Brasil essa assistência adequada as mulheres grávidas, visto que o país ainda preza por uma medicina hospitalocêntrica, tecnocrática e medicalizante (BRASIL, 2012).

Dessa forma, o pré-natal é essencial para manutenção e melhoria da saúde das mulheres e crianças e deve ser fornecida pelo Sistema Único de Saúde (SUS), seguindo as recomendações estabelecidas pelo Ministério da Saúde (MS) (FONSECA MRCC, et al., 2019). O MS afirma que para o pré-natal ser de qualidade as mulheres gravidas devem ser acompanhadas durante toda a sua gravidez e no puerpério, com o objetivo de garantir o nascimento de um recém-nascido saudável e também proporcionando garantir o bem-estar da puérpera e do filho (TOMASI E, et al., 2017).

O pré-natal de mulheres sem complicações durante o curso da gravidez é chamado Pré-Natal de Baixo Risco, sendo realizado na Unidade Básica de Saúde (UBS), que deve configurar-se como a porta de entrada da gestante ao sistema de saúde (BRASIL, 2012). O fornecimento universal do pré-natal é estabelecido como um direito da mulher, e garante maior qualidade à gestação, ao parto e ao pós-parto. Muitos fatores podem contribuir para os desfechos perinatais, sendo a assistência pré-natal uma condição que pode contribuir para melhorar esses desfechos, realizando diagnósticos e tratamentos oportunos, e atuando nos fatores de risco que podem afetar a saúde materna e do recém-nascido (DOMINGUES RMSM, et al., 2012).

Além disso, o baixo número de consultas pré-natais pode se configurar como um fator de risco para mortalidade fetal e neonatal, e que se as intervenções não são feitas em momentos adequados, pode ocasionar nascimento prematuro (MARTINELLI KG, et al., 2014). No Brasil, por volta da década de 80, houve a implantação do Programa de Atenção Integral à Saúde da Mulher (PAISM), no qual se evidenciou uma propagação de forma mais abrangente da atenção pré-natal. E, a partir de então, progressivamente notou-se a melhora de indicadores de qualidade do pré-natal como a cobertura, o número e o início das consultas (PAIVA CCND e CAETANO R, 2020).

Em 2000 foi instituído pelo MS o Programa de Humanização no Pré-natal e Nascimento (PHPN), que regulamentou o pré-natal, estabelecendo um acesso digno e de qualidade aos serviços e ações, de forma a 
garantir um pré-natal, parto e puerpério humanizados e de qualidade. Nesse programa foi definido, dentre outras condutas, quando deve se iniciar o pré-natal e quantas consultas devem ser realizadas, e foram instituídos os exames laboratoriais básicos que precisam ser solicitados, a necessidade da realização da imunização antitetânica, realização de atividades educativas e classificação de risco gestacional em todas as consultas (BRASIL, 2011).

Ademais, contribuindo para uma maior cobertura pré-natal, em 2006 a Estratégia Saúde da Família foi firmada como o modelo que reorganizou a atenção primária à saúde e sua expansão no Brasil, assim como a implantação do PHPN, foram estratégias adotadas para reduzir a mortalidade materna no Brasil (ANVERSA ETR, et al., 2012).

Outro programa de destaque é a Rede Cegonha, implantada em 2011, que se constitui como uma rede de cuidados para assegurar o direito ao planejamento reprodutivo, conduzir a gravidez, parto e puerpério de modo a possibilitar um nascimento seguro da criança, inclusive abarcando seu crescimento e desenvolvimento saudáveis. Essa rede é formada por quatro componentes, sendo um deles o pré-natal (BRASIL, 2011). Essa evolução histórica do pré-natal no Brasil, com a implantação dos programas supracitados, oportunizou um maior acesso a serviços de saúde necessários no período gestacional, objetivando qualidade na assistência às mulheres e seus filhos (BRASIL, 2011).

Entretanto, é válido ressaltar que, para demonstrar a efetividade de um programa de saúde, é necessário avaliar o seu processo, pois ele permite analisar os cuidados que são realmente proporcionados e recebidos pelos usuários durante a assistência (BALSELLS MMD, et al., 2018). Consequentemente, torna-se um importante instrumento para direcionar gestores e profissionais da saúde, de modo que eles possam atuar de forma mais assertiva nos pontos do sistema que devem ser fortalecidos (ANVERSA ETR, et al., 2012).

Dessa forma, o objetivo desse trabalho foi avaliar a assistência pré-natal ofertada às gestantes de Vitória da Conquista - BA durante o ano de 2019, orientando-se pelos tópicos contemplados nos indicadores de processo estabelecidos pelo PHPN, que atualmente ainda são utilizados como base para a avaliação da oferta do pré-natal no Brasil.

\section{MÉTODOS}

Esta pesquisa possui caráter descritivo, com natureza e abordagem quantitativa. Os dados secundários de domínio público que foram analisados derivam de prontuários eletrônicos que alimentam o sistema e-SUS $A B$, portanto, o critério de inclusão dessa pesquisa contempla mulheres que tiveram seus registros cadastrados nesses prontuários no ano de 2019, e os critérios de exclusão foram informações que derivam de prontuários físicos, além de consultas pré-natais realizadas em hospitais e clínicas privadas. Foi obtida uma amostra total de 5486 mulheres gestantes durante o ano de 2019 em Vitória da Conquista que tiveram seus prontuários preenchidos na época do pré-natal.

A Estratégia e-SUS AB, foi adotada pelo Ministério da Saúde em 2013 como uma nova proposta de sistema de informação, com o objetivo de qualificar e viabilizar as informações coletadas na Atenção Básica. Essa Estratégia tem o intuito de diminuir o trabalho dos profissionais de saúde através de um modelo integrado de registro de informações, sendo criada uma única entrada de dados para os serviços de Atenção Básica, buscando evitar que os profissionais de saúde tenham que acessar vários sistemas ao mesmo tempo (ARAÚJO JRD, et al., 2019).

Sua implantação aconteceu de forma gradual e, a partir de 2017, o Sistema de Acompanhamento da Gestante que até então configurava-se como um sistema nacional e independente para armazenar dados referentes ao pré-natal das gestantes, começou a ser substituído pelo e-SUS AB. As Unidades Básicas de Saúde enviam regularmente informação para o e-SUS AB e nesse sistema ficam registrados os dados de pré-natal e puerpério provenientes dos serviços da Atenção Básica (BRASIL, 2017). Portanto, as informações presentes nesse trabalho derivam de registros municipais coletados no sistema online e-SUS AB.

Para selecionar as variáveis e verificar a adequação do pré-natal, utilizou-se como base, e de forma adaptada, os indicadores de processo estabelecidos pelo PHPN. Foram analisados os seguintes tópicos: 
Número de consultas pré-natais de cada gestante, solicitação de exames básicos necessários nesse período e a realização da consulta puerperal. Após consolidar os dados, os mesmos, foram analisados e organizados sistematicamente em tabelas e gráfico por meio da utilização do programa Microsoft Excel $₫ 2016$.

\section{RESULTADOS E DISCUSSÃO}

Foram analisados 5486 prontuários eletrônicos de gestantes em relação a consultas realizadas. As informações demostram que $3,5 \%$ das gestantes não realizaram nenhuma consulta pré-natal, $7,5 \%$ realizaram de uma a três consultas, $30,93 \%$ realizaram de quatro a seis consultas e mais da metade $(58,42 \%)$ realizaram sete ou mais consultas pré-natais (Tabela 1). A porcentagem de mulheres que realizaram sete ou mais consultas, foi menor do que a encontrada em estudo prévio realizado em outra cidade no Nordeste, no qual observou-se que 60,1\% das mulheres analisadas atingiram essa marca (SILVA EPD, et al., 2019).

Tabela 1 - Número de gestantes no ano de 2019 em relação ao número de consultas realizadas durante o pré-natal. Vitória da Conquista, Bahia. $(\mathrm{n}=5486)$.

\begin{tabular}{ccc}
\hline Número de consultas & "n" & $\%$ \\
\hline zero & 192 & 3,5 \\
$1-3$ & 392 & 7,15 \\
$4-6$ & 1697 & 30,93 \\
7 ou mais & 3205 & 58,42 \\
\hline Total & 5486 & 100 \\
\hline
\end{tabular}

Fonte: Poloni APB, et al., 2021.

A média de consultas por gestante foi de 5,77, posicionando-se como uma média inferior as encontradas em diversos estudos $(5,86 ; 6,2 ; 6,6 ; 6,8)$ (ANVERSA ETR, et al., 2012). Foi demonstrado também que 96,5\% das gestantes estiveram presentes em, pelo menos, uma consulta, número maior do que o visto em uma pesquisa realizada na cidade do Maranhão, que registrou a porcentagem de 89,5\% (BRASIL TB, et al., 2018). Em outro estudo mais recente, também no estado do Maranhão, a inferioridade dos dados se repetiu, onde foi demonstrado que $86 \%$ das gestantes realizaram pelo menos uma consulta pré-natal (COSTA GRC, et al., 2010).

Outro ponto analisado foi a realização de exames básicos no pré-natal. A Tabela 2 mostra dados referentes a 903 consultas pré-natais registradas no sistema, e lista os exames que devem ser solicitados na primeira consulta pré-natal, e entre eles, alguns que devem ser repetidos no terceiro trimestre de gestação. Além disso, informa o número de solicitações que esses exames registraram no e-SUS AB durante a rotina do pré-natal nas Unidades de Saúde em 2019.

Tabela 2 - Relação de exames solicitados durante 2019 em 903 consultas pré-natais realizadas nas Unidades de Saúde de Vitória da Conquista, Bahia $(n=903)$.

\begin{tabular}{ccc}
\hline Examessolicitados & "n" & $\%$ \\
\hline Hemograma* $^{*}$ & 828 & 91,69 \\
\hline Tipagemsanguínea & 189 & 20,93 \\
\hline PesquisadofatorRH & 208 & 23,03 \\
\hline Glicemiadejejum & 89,81 \\
\hline Testerápidoparasífilise/ouVDRL $^{*} / \mathrm{PRP}$ & 803 & 88,92 \\
\hline TesterápidoparaHIV & 23 & 2,54 \\
\hline Anti-HIV* & 791 & 87,59 \\
\hline ToxoplasmoselgM $^{*}$ & 796 & 88,15 \\
\hline ToxoplasmoselgG & 649 & 71,87 \\
\hline SorologiaparahepatiteB(HbsAg) $^{*}$ & 670 & 74,19 \\
\hline Examedeurina $^{*}$ & 830 & 91,91 \\
\hline Urocultura* $^{*}$ & 680 & 75,30
\end{tabular}

Legenda: *Exames que além de solicitados na primeira consulta, precisam ser repetidos no 3 o trimestre. Se toxoplasmose IgG não reagente no primeiro resultado, repetir no $3^{0}$ trimestre também os exames de toxoplasmose. Fonte: Poloni APB, et al., 2021. 
Em relação ao hemograma, foram solicitados 828 exames nas consultas pré-natais analisadas. A tipagem sanguínea e pesquisa de fator $\mathrm{RH}$ foram feitas 189 e 208 vezes, respectivamente. A glicemia em jejum, foi solicitada 811 vezes, resultado próximo ao da solicitação do teste rápido para sífilis e/ou VDRL/PRP, que foi de 803.

Em relação ao diagnóstico de HIV, foram encontradas 23 solicitações de teste rápido e 791 de teste antiHIV. Os exames para detecção de toxoplasmose foram registrados separados, obtendo 796 solicitações de toxoplasmose IgM e 649 de toxoplasmose $\mathrm{lgG}$. A sorologia para hepatite B ( $\mathrm{Hbs} A g$ ) foi solicitada 670 vezes e, por fim, o exame de urina (EAS) teve 830 solicitações e o exame de urocultura contabilizou 680 solicitações.

Portanto, percebe-se que os exames que mais solicitados, nesta ordem, foram: exame de urina $(91,91 \%)$ e hemograma (91,69\%). Esse fato, em parte se assemelha a resultados encontrados quando se avaliou a qualidade da atenção pré-natal na rede básica de saúde do Brasil, em que um dos exames mais solicitados também foi o exame de urina (TOMASI E, et al., 2017). Porém, demonstra valores inferiores aos encontrados na solicitação de exames de sangue e urina na rede SUS na cidade do Rio de Janeiro, que atingiu $98 \%$ das gestantes (DOMINGUES RMSM, et al., 2012).

Os exames menos solicitados foram: teste rápido para HIV (2,54\%), tipagem sanguínea $(20,93 \%)$ e pesquisa do fator $\mathrm{RH}(23,03 \%)$. O baixo valor encontrado para a realização de teste rápido para HIV também foi presente em uma análise realizada em uma cidade do Espírito Santo (13,4\%), porém, nesse mesmo estudo, os resultados da realização de tipagem sanguínea e fator $\mathrm{RH}$ foram mais animadores, atingindo $88,6 \%$ da amostra analisada (MARTINELLI KG, et al., 2014).

Em relação ao número de consultas puerperais efetuadas, a porcentagem de realização dessa consulta foi obtida estabelecendo como base o número de nascidos vivos no ano de 2019 ( $n=5541)$, pois essa variável relaciona-se mais fielmente com a quantidade de mulheres que deveriam realizar a consulta puerperal do que a amostra do número total de gestantes em 2019. O Gráfico 1 informa que apenas $23,78 \%(n=1318)$ das gestantes realizaram a consulta puerperal.

Esse resultado é consideravelmente menor do que os obtidos em outros estudos. Em uma pesquisa realizada no Centro-Oeste $43,8 \%$ das mulheres compareceram a consulta puerperal (FUSQUINE RS, et al., 2019), em outro estudo, na região Sudeste, a consulta puerperal foi realizada em $58,7 \%$ das gestantes e, no Sul no Brasil, o índice foi ainda melhor, atingindo 75,2\% das puérperas (TSUNECHIRO MA, et al., 2018; GONCALVES CDS, et al., 2019).

Gráfico 1 - Número de consultas puerperais realizadas em 2019, em relação ao número de nascidos vivos no mesmo ano em Vitória da Conquista, Bahia $(\mathrm{n}=5541)$.

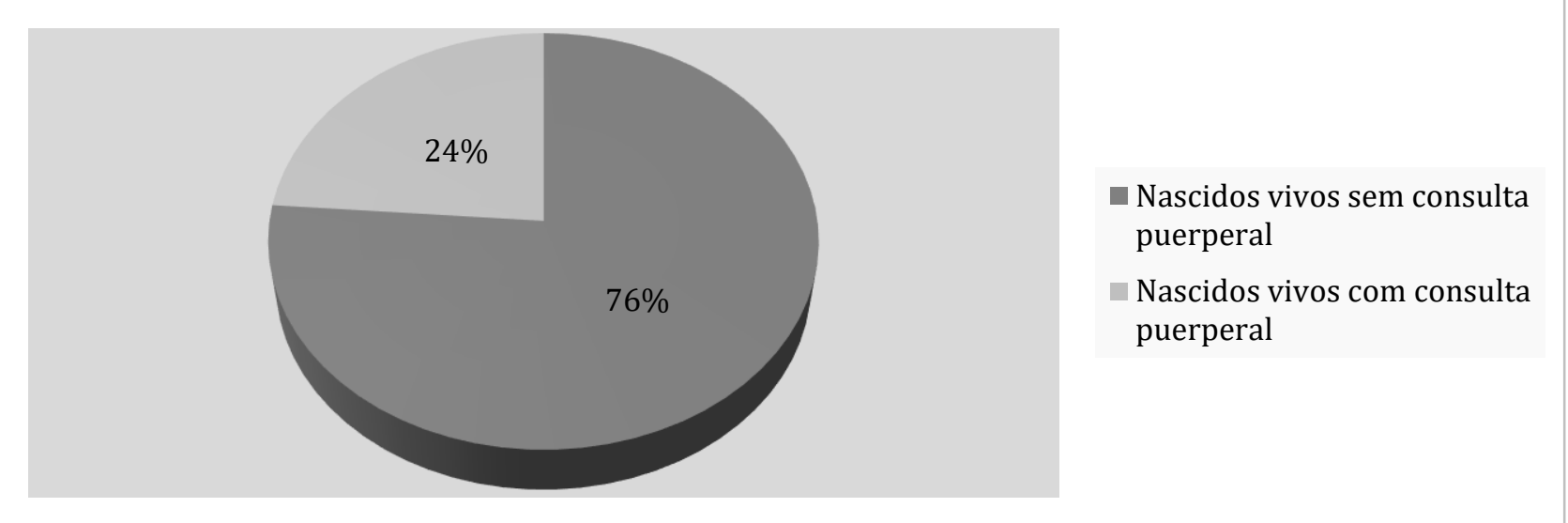

Fonte: Poloni APB, et al., 2021.

Não foi possível obter os dados referentes a época de início do pré-natal das gestantes e sobre a imunização antitetânica nas gestantes em 2019. Tal fato constituiu-se como um empecilho para uma análise 
mais detalhada e completa sobre a situação da assistência pré-natal em Vitória da Conquista, limitando a verificação de variáveis importantes relacionadas a esse tema.

Por mais que identificar a época de início do pré-natal seja de extrema importância para avaliar a assistência pré-natal ofertada, o número de consultas realizadas por gestante pode refletir o início do prénatal, pois quanto mais precoce for a primeira consulta, a qualidade da assistência será melhor e isso, consequentemente, resultará em um número maior de consultas (COSTA GRC, et al., 2010).

Os dados obtidos na pesquisa, no que tange as consultas pré-natais, revelam que o município de Vitória da Conquista apresentou uma média de consultas por mulher abaixo do que o preconizado pelo MS, pois o "Caderno de Atenção Básica: Atenção ao Pré-natal de Baixo Risco", documento que define diversas condutas que devem ser seguidas durante o pré-natal na Atenção Básica, recomenda o mínimo de seis consultas prénatais (BRASIL, 2012).

No entanto, a porcentagem de mulheres que realizaram pelo menos uma consulta pré-natal foi superior as analisadas em outros estudos, fato que indica que a maioria das mulheres estão conseguindo acessar 0 sistema, tornando-se evidente, dessa forma, a necessidade de ser fortalecido o acompanhamento e vínculo com essa gestante, para que ela prossiga na realização do pré-natal durante toda a gestação.

Em relação aos exames que devem ser solicitados durante o pré-natal, observou-se um déficit considerável em solicitações de exames básicos e necessários. Dentre eles, chama atenção a baixa porcentagem de mulheres que realizaram teste rápido para HIV, tipagem sanguínea e pesquisa de fator $\mathrm{RH}$. Esses são exames que devem ser solicitados, inclusive na primeira consulta pré-natal, e obtê-los auxilia para o progresso de uma gestação e parto mais saudáveis e seguros, contribuindo diretamente para uma assistência pré-natal de maior qualidade.

No entanto, uma ressalva deve ser pontuada no que se refere a baixa realização do teste rápido para HIV. Mesmo a solicitação desse teste sendo apontada como necessária na primeira consulta, esse resultado, que revela sua baixa realização, pode não refletir uma grave defasagem na identificação do vírus HIV nas grávidas de Vitória da Conquista, pois outro teste diagnóstico e necessário, o teste ANTI-HIV, apareceu em 87,59\% das solicitações.

Por conseguinte, observando a realização da consulta puerperal, nota-se uma falha no acompanhamento das gestantes, pois é recomendado que, dentro da Atenção Básica, toda mulher realize sua consulta puerperal. Diante disso, foi constatado que apenas $23,78 \%$ dessas consultas foram realizadas, assim sendo, nem um terço das gestantes foram alcançadas pelo sistema de saúde para esse serviço.

Um pré-natal é considerado completo após a realização da consulta puerperal porque sua realização define condutas para garantir o adequado período pós-parto, que protege a mulher e orienta sobre questões como a introdução de métodos contraceptivos, além de diagnosticar alterações como anemia e estados depressivo (CRUZ GCD, et al., 2019). Ademais essa atenção especial a puérpera ajuda na adaptação à condição materna e auxilia no cuidado do filho frágil e dependente, pois inclui o desenvolvimento de habilidades necessárias para essa mãe (PEREIRA MC e GRADIM CVC, 2014).

Outro aspecto que torna a consulta puerperal essencial é a possibilidade de realizar a triagem neonatal, conferir se o recém-nascido foi imunizado com as vacinas BCG e Hepatite B e observar o aleitamento materno, para, se necessário, oferecer orientações e apoio. Essas ações são preconizadas pela Rede Cegonha por meio da estratégia "Primeira Semana de Saúde Integral" (VILELA MLF e PEREIRA QLC, 2018). Dessa forma é necessário aumentar a cobertura da consulta puerperal, para uma maior efetividade nesse quesito que faz parte do pré-natal e que define a qualidade do acompanhamento da gestante.

Baseando-se nos dados colhidos, nota-se que a assistência pré-natal em Vitória da Conquista ainda não atingiu sua excelência, persistindo gestantes que não tiveram acesso à consulta pré-natal e, poucas realizaram todos os exames necessários, além da média de consultas por gestante ser inferior ao ideal e apresentar uma baixa taxa de consultas puerperais. Dessa forma, é necessário fortalecer a assistência prénatal nesta cidade do Sudoeste baiano, por meio de ações que garantam a captação e o acompanhamento de forma mais efetiva das gestantes, inclusive após o parto. 
Em relação os exames realizados, limitações no fornecimento de dados pelo sistema não informaram de maneira detalhada quais exames cada mulher realizou e se eles foram solicitados mais de uma vez para a mesma gestante, só foi possível observar as solicitações de consultas pré-natais de forma geral. Apesar de tal circunstância, os resultados obtidos são válidos e importantes de serem discutidos, pois os números encontrados verificam ainda uma baixa abrangência na realização de todos os exames básicos do pré-natal.

Por fim, sobre as consultas puerperais, foi notado a necessidade da evolução da atenção puerperal, uma vez que os resultados apontam não contemplarem a maioria das puérperas, consequentemente refletindo uma baixíssima taxa de adequação do acompanhamento pré-natal, pois essa consulta configura-se como um dos pontos indispensáveis a serem cumpridos pela Atenção Básica.

\section{CONCLUSÃO}

Em resumo, a assistência pré-natal às gestantes de Vitória da Conquista apresentou baixa adequação e ainda necessita de melhorias em diversos aspectos. Além de não garantir o acompanhamento pré-natal a todas as mulheres que estiveram grávidas durante o ano de 2019, a média de consultas por mulher não atingiu a marca mínima estabelecida pelo MS, que é de seis consultas. Por fim, avaliar como o pré-natal vem sendo oferecido a mulher é indispensável para indicar quais aspectos da assistência precisam ser aperfeiçoados e pode configurar-se como um estímulo para gestores e profissionais da saúde atuarem nas deficiências identificadas. Desse modo, pesquisas como esta visam possibilitar uma reflexão sobre a assistência prestada às gestantes, recém-nascidos, suas famílias e à sociedade, análise dos indicadores de saúde e intervenções de políticas públicas eficientes para acompanhamento ao pré-natal e puerpério de qualidade.

\section{REFERÊNCIAS}

1. ARAÚJO JRD, et al. Sistema e-SUS AB: percepções dos enfermeiros da Estratégia Saúde da Família. Rev. Saúde Pública, 2019; 43(122): 780-792.

2. ANVERSA ETR, et al. Qualidade do processo da assistência pré-natal: Unidades Básicas de Saúde e Unidades de Estratégia Saúde da Família em município no Sul do Brasil. Cad. Saúde Pública, 2012; 28(4): 789-800.

3. BRASIL. Ministério da Saúde. Nota Técnica ํo 19 de 30 de novembro de 2017. Esclarece sobre a utilização do eSUS AB e Sisprenatal Web no âmbito da Atenção Básica. 2017. Disponível em: http://www.saude.ba.gov.br/wpcontent/uploads/2018/08/NOTA-T\%C3\%89CNICA-SISPRENATAL-N\%C2\%BA-19-SEI-2017-CGSMU-DAPES-SASMS.pdf. Acessado em: 8 de outubro de 2020.

4. BRASIL. Ministério da Saúde. Secretaria de Atenção à Saúde. Departamento de Atenção Básica. Cadernos de Atenção Básica: Atenção ao Pré-natal de Baixo Risco. 2012 . Disponível em: https://aps.saude.gov.br/biblioteca/visualizar/MTIwOQ. Acessado em: 8 de outubro de 2020.

5. BRASIL. Ministério da Saúde. Gabinete do Ministro. Portaria no. 1459, de 24 de junho de 2011. Institui a Rede Cegonha. 2011. Disponível em: http://bvsms.saude.gov.br/bvs/saudelegis/gm/2011/prt1459_24_06_2011.html. Acessado em: 8 de outubro de 2020.

6. BRASIL TB, et al. Fatores associados à mortalidade neonatal com ênfase no componente da atenção hospitalar ao recém-nascido. Arquivos Catarinenses de Medicina, 2018; 47(2): 70-86.

7. BALSELLS MMD, et al. Avaliação do processo na assistência pré-natal de gestantes com risco habitual. Acta Paulista de Enfermagem, 2018; 31(3): 247-254.

8. COSTA GRC, et al. Caracterização da cobertura do pré-natal no Estado do Maranhão, Brasil. Rev. Bras. Enferm.,2010; 63(6): 1005-1009.

9. CRUZ GCD, et al. Métodos de avaliação da qualidade de assistência ao pré-natal no Brasil: revisão integrativa da literatura. Revista Eletrônica Acervo Saúde, 2019; 27:e521.

10. DOMINGUES RMSM, et al. Avaliação da adequação da assistência pré-natal na rede SUS do Município do Rio de Janeiro, Brasil. Cad. Saúde Pública, 2012; 28(3): 425-437.

11. FUSQUINE RS, et al. Adesão e rejeição à consulta puerperal por mulheres de uma unidade básica de saúde da família. Arquivos de Ciências da Saúde, 2019; 26(1): 37-40.

12. FERREIRA TLDS, et al. Avaliação da assistência com foco na consulta de atendimento pré-natal. Revista Ciência Plural, 2017; 3(2): 4-15.

13. FONSECA MRCC, et al. Perfil sociodemográfico e acesso à assistência pré-natal das puérperas de um hospital público. Revista Família, Ciclos de Vida e Saúde no Contexto Social, 2019; 7(1): 6-15.

14. GONCALVES CDS, et al. Frequência e fatores associados à não realização da consulta puerperal em um estudo de coorte. Rev. Bras. Saúde Mater. Infant., 2019; 19(1): 63-70. 
15. MARTINELLI KG, et al. Adequação do processo da assistência pré-natal segundo os critérios do Programa de Humanização do Pré-natal e Nascimento e Rede Cegonha. Rev. Bras. Ginecol. Obstet., 2014; 36(2): 56-64.

16. PEREIRA MC, GRADIM CVC. Consulta puerperal: a visão do enfermeiro e da puérpera. Ciência, Cuidado e Saúde, 2014; 13(1): 35-42.

17. PAIVA CCND, CAETANO R. Avaliação de implantação das ações de saúde sexual e reprodutiva na Atenção Primária: revisão de escopo. Escola Anna Nery, 2020; 24(1): e20190142.

18. SILVA EPD, et al. Avaliação do pré-natal na atenção primária no Nordeste do Brasil: fatores associados à sua adequação. Rev. Saúde Pública, 2019; 53(43): 173-179.

19. TSUNECHIRO MA, et al. Avaliação da assistência pré-natal conforme o Programa de Humanização do Pré-Natal e Nascimento. Revista Brasileira de Saúde Materno Infantil, 2018; 18(4): 771-780.

20. TOMASI E, et al. Qualidade da atenção pré-natal na rede básica de saúde do Brasil: indicadores e desigualdades sociais. Cad. Saúde Pública, 2017; 33(3): e00195815.

21. VILELA MLF, PEREIRA QLC. Consulta puerperal: orientação sobre sua importância. J. Health NPEPS, 2018; 3(1): 228-240. 